\title{
Optical properties of impact diamonds from the Popigai astrobleme
}

\author{
A. Yelisseyev ${ }^{\text {a, }}$, G.S. Meng b,e ${ }^{\text {, }}$ V. Afanasyev ${ }^{\text {a }}$, N. Pokhilenko ${ }^{a}$, V. Pustovarov ${ }^{c}$, A. Isakova a , \\ Z.S. Lin ${ }^{\text {b,* }}$, H.Q. Lin $^{\mathrm{d}}$ \\ a Institute of Geology and Mineralogy, Russian Academy of Sciences, Siberian Branch, 3 Academician Koptyug Ave., Novosibirsk 630090, Russia \\ ${ }^{\mathrm{b}}$ BCCRD, Key Laboratory of Functional Crystals and Laser Technology, Technical Institute of Physics and Chemistry, CAS, P.0. Box 2711, Beijing 100190, China \\ c Ural Federal University, 19 Mira Str., Yekaterinburg 620002, Russia \\ d Beijing Computational Science Research Center, No. 3 He-Qing Road, Hai-Dian District, Beijing 100084, China \\ e Key Laboratory of Quantum Information, University of Science and Technology of China, Chinese Academy of Sciences, Hefei, Anhui 230026, China
}

\section{A R T I C L E I N F O}

\section{Article history:}

Received 8 January 2013

Received in revised form 7 April 2013

Accepted 15 April 2013

Available online 30 April 2013

\section{Keywords:}

Carbon polytypes

Impact nanostructures

Optical properties

Superhard materials

\begin{abstract}
A B S T R A C T
Impact diamonds from Popigai astrobleme were found to consist of different carbon phases: cubic and hexagonal diamond with $s p^{3}$ bonding according to X-ray structural analysis as well as amorphous, crystalline and disordered graphite with $s p^{2}$-bonding (Raman scattering). The sizes of graphite domains vary from 10 to $100 \mathrm{~nm}$. Fundamental absorption edge for Popigai impact diamonds is shifted $\sim 0.5 \mathrm{eV}$ to lower energies in comparison with kimberlite diamonds $(5.47 \mathrm{eV})$ as a result of the lonsdaleite input, in good agreement with ab initio calculations ( $E_{g}=5.34$ and $4.55 \mathrm{eV}$ for $3 \mathrm{C}$ cubic and $2 \mathrm{H}$ hexagonal diamonds, respectively). Yellowish color of impact diamonds is due to Rayleigh light scattering on structural defects whereas graphite is responsible for gray to black coloring. In the mid-IR region there is a multi-phonon absorption of $3 \mathrm{C}$ diamond in the 1800 to $2800 \mathrm{~cm}^{-1}$ range and some new bands at $969,1102,1225$, and $1330 \mathrm{~cm}^{-1}$ in the one-phonon region. Micro-Raman study shows inclusions of side noncarbon minerals (quartz, magnetite, and hematite) some of which contain $\mathrm{Cr}^{3+}$ impurity. The vibration modes of cubic diamond and lonsdaleite exhibited in the Raman spectra were elucidated by the first-principles studies. Popigai impact diamonds demonstrate a broad-band luminescence in 2.1,2.38, and $2.84 \mathrm{eV}$ components similar to that for nanocrystal polycrystalline $3 \mathrm{C}$ diamond. All emissions are excited at band-to-band transitions whereas the last two are observed also at excitation into 2.4 and 3.0 bands supposedly as a result of intracenter processes within the $\mathrm{H} 3(\mathrm{NVN})$ and $\mathrm{NV}^{0}$ centers.
\end{abstract}

(c) 2013 Elsevier B.V. All rights reserved.

\section{Introduction}

For a long time diamonds attract attention by their unique parameters: the record chemical, mechanical and radiation stability, thermal conductivity and mobility of electrons and holes among the solids. Diamonds are known to form deep in the Earth at high pressures and temperatures $\left(\mathrm{P}>4.5 \mathrm{GPa}, \mathrm{T}>1000{ }^{\circ} \mathrm{C}\right)$ in natural conditions and they are brought later to the Earth surface. Today diamond is one of the most studied inorganic materials and nanocrystalline diamond, thin diamond films and high quality bulk crystals are produced in the laboratory/ industrial conditions for different applications in gemology, science and industry. Much less is known about impact diamonds, which form in the shock process when a meteorite falls on the Earth [1]. As early as 1888, impact diamonds were found by Erofeev and Lachinov in the meteorite from the Novo Urei, Russia [2], and later in the meteorite from Canyon Diablo in Arizona [3] and from many other craters such as Popigai astrobleme (Russia), Ries (Germany), Kara and Puchezh-Katunski (Russia) [4,5].

\footnotetext{
* Corresponding authors.

E-mail addresses: eliseev.ap@mail.ru (A. Yelisseyev), zslin@mail.ipc.ac.cn (Z.S. Lin).
}

Recently, the evidences of shock-synthesized impact diamonds were investigated to correlate with the abrupt ecosystem change and megafaunal extinction triggered by a cosmic impact (for example, over North America about 13,000 years ago) [6].

The impact diamonds were derived at extremely high P-T parameters (temperature up to $4000{ }^{\circ} \mathrm{C}$ and pressure up to $140 \mathrm{GPa}$ ) from either parental graphite or coal as a result of solid state phase transition [5]. This conclusion is based on the analysis of the grain morphology, the preferred orientation of crystallites, the occurrence of lonsdaleite (a high-pressure polymorph of carbon with hexagonal close-packed structure) associated with target rock inclusions (material exposed at a site of an impact before crater formation), and the occurrence within large bodies of impact melt rocks. Investigation of meteorite diamonds allowed one to identify a set of new $s p^{3}$ carbon phases both of hexagonal $(4 \mathrm{H}, 6 \mathrm{H}, 8 \mathrm{H}, 10 \mathrm{H}-1$ and $10 \mathrm{H}-2)$ and rhombohedral (15R and $21 \mathrm{R})$ symmetry [7]. These crystalline forms together with cubic (3C) diamond and lonsdaleite (2H hexagonal diamond) were called diamond polytypes [7]. The diamond polytypes were identified by infrared and Raman spectroscopy as well as electron and X-ray diffraction [7]. To date only cubic $3 C$ diamond and lonsdaleite in these $s p^{3}$ polytypes together with graphite $\left(s p^{2}\right)$, chaoite $\left(s p^{1}\right)$ and carbides namely $\mathrm{SiC}$ 
have been found in terrestrial impact diamonds, and the content of lonsdaleite reaches $80 \%$ sometimes [5].

The cubic diamonds can be grown by tangential or normal mechanisms depending on the degree of melt supersaturation: as a result the crystals with octahedral or cubic habit are obtained [8]. For cubic diamonds with octahedral habit the growth on a seed is very slow and layer by layer mechanism takes place. So one needs hundreds of hours in laboratory conditions and much longer time, in geological scale, in nature. In the case of cubic habit, a multicenter nucleation process takes place, and many crystals as needles are growing simultaneously and competing with each other. One can usually observe the spherulite structures inside such diamonds. However, in a shock wave the formation of the impact diamonds is a considerably different process of solid state phase transition [9]. The shock compression acting on the graphite grains makes the lonsdaleitediamond paramorphs consist of very small crystallites. According to $\mathrm{X}$-ray structural analysis the effective radius $\mathrm{L}$ of such crystallite varies from $10 \mathrm{~nm}$ to $100 \mathrm{~nm}$, L for lonsdaleite being smaller than that for cubic $3 \mathrm{C}$ diamond [5].

The Popigai astrobleme in the north of Siberia in Russia is one of the largest craters all over the world: its diameter approaches to about $100 \mathrm{~km}$. The remarkable feature of this crater is a high content of graphite in the breeds: as a result a lot of impact diamonds formed during the impact event and their reserves exceed many times those of kimberlite diamonds all over the world. Our experimental estimations showed that the wear hardness of impact diamonds from the Popigai crater exceeds 2 to 2.5 times that of the cubic $3 C$ diamonds, thus this material is of great interest for industrial applications. On one hand, high resistance to wear for Popigai impact diamonds (PID) may be a result of the lonsdaleite presence in the structure. The lonsdaleite was reported to be an extremely hard material, whose hardness was estimated to be 1.58 higher compared to cubic diamond [10]. On the other hand, it may be a result of PID nanocrystalline structure as in the case of $3 \mathrm{C}$ nano-polycrystalline diamonds [11]. Till now there is very limited information about optical properties of impact diamonds which could be important also for optoelectronics. There are only a very few measures on the cathodoluminescence $(\mathrm{CL})$ and photoluminescence (PL) properties of impact diamonds, which revealed that the broad emission bands dominate in the visible CL [12] and PL [5] spectra although there is also some fine structure [5]. Only N3 vibronic system corresponding to the nitrogen-vacancy complex $\mathrm{N}_{3} \mathrm{~V}$ in cubic 3C diamonds [13] was identified in PID samples [5]. To date there is no information about the transmission spectra of impact diamonds: it may be a result of their small size, complicated shape, intense color as well as the difficulty of sample preparing from such superhard material.

In present paper the phase composition of impact diamonds from the Popigai astrobleme (IDP) was studied using the X-ray structural analysis and Raman spectroscopy: $3 \mathrm{C}$ cubic and $2 \mathrm{H}$ hexagonal diamond, graphite were found to be dominating phases with $20 \%$ lonsdaleite input plus some noncarbon mineral phases. No nitrogen related signal was observed in one-phonon range, but strong absorption occurred from pressurized solid carbon dioxide $\left(\mathrm{CO}_{2}\right)$. Raman spectroscopy confirms the presence of the nanometer-sized structural domains although PID plate interacts as a monolithic media with a mid-IR light wave with $\lambda \sim 5-10 \mu \mathrm{m}$. The experimental peaks of Raman spectra are identified and their lattice vibrational origins are elucidated by the first-principles studies. Transmission spectra in the UV to mid IR range were recorded for PID and compared with the ab initio estimation of band gaps: $E_{g}$ for lonsdaleite was found to be $0.5 \mathrm{eV}$ smaller in comparison with cubic diamond. Furthermore, low-temperature PL and PL excitation spectra of the samples were measured using the synchrotron radiation. Spectroscopic features of impact diamonds are different considerably from those of natural kimberlite bulk diamonds but they are similar to the features of polycrystalline $3 \mathrm{C}$ nanodiamonds.

\section{Experimental and computational methods}

\subsection{Samples}

Several hundreds of impact diamonds were separated by thermochemical (alkaline fusion) treatment of impact melt rocks from the Popigai astrobleme following the procedure of Rost et al. [14]. The PID samples have relatively small grains with the average size of $1.5 \mathrm{~mm}$ and exhibit various colors, ranging from pale-yellow to yellow, yellowish-gray, gray, brownish-gray, and black (Fig. 1(a)). For a batch of transparent samples we obtained the X-ray diffraction patterns using a powder diffractometer (ARL X'TRA from the Thermo Scientific) operating at the $\mathrm{CuK} \alpha$ radiation (see Fig. S1 in the Supplementary data). It is demonstrated that the dominating carbon phases in PID are nanometer-sized cubic (3C) and hexagonal (2H) diamonds and the content of the latter does not exceed $20 \%$. Val'ter et al. found that in the strongly colored or black PID samples the lonsdaleite input is larger, reaching $80 \%$ [5].

We studied optical absorption and photoluminescence in several tens of PID samples, which were transparent, slightly colored plates with intense yellow photoluminescence at room temperature at UV $365 \mathrm{~nm} \mathrm{Hg}$ excitation. One of these impact diamonds (G4) is shown in Fig. 1(b)-(f). One can see that it is a plate $\sim 112 \mu \mathrm{m}$ thick with well-pronounced flat and parallel opposite faces (Fig. 1(d)). This sample demonstrates a yellow PL, which is typical of all examined impact diamonds. Deeper colored PID samples have a weaker PL. In polarized transmitted light one can see a variety of colors with a lot of finesized details, which indicates a complicated, polycrystalline structure of the sample. Gray or black color of PID is due to graphite inclusions. To clean the sample surface before spectroscopic measurements impact diamonds were boiled $10 \mathrm{~min}$ in chloronitric acid (a 3:1 mixture of hydrochloric and nitric acids), which allowed us to remove organic and mineral contaminations from the developed surface of the samples.

\subsection{Spectroscopic studies}

Transmission spectra were recorded using a UV-2501PC Shimadzu spectrometer in the UV- to near-IR, whereas in the mid-IR we used a Fourier-Transform spectrometer Infralum 801. For local measurements we used a FTIR spectrometer Vertex 70 combined with a microscope Hyperion 2000. Typical diameter of the beam was 50 to $100 \mu \mathrm{m}$.

Raman spectra were recorded with spectral resolution of $1 \mathrm{~cm}^{-1}$ at room temperature at excitations $514.5 \mathrm{~nm}\left(\mathrm{Ar}^{+}\right.$laser $)$and $325 \mathrm{~nm}$ (He-Cd laser) using a confocal LabRam micro-Raman spectrometer. The low-temperature PL and PLE spectra were measured at $7.5 \mathrm{~K}$ using synchrotron radiation on a SUPERLUM station of the DESYLAB laboratory (Hamburg, Germany) [15]. The PLE spectra were measured at excitation energy $E_{\mathrm{ex}}=4$ to $40 \mathrm{eV}$ and normalized to an equal number of incident photons using the sodium salicylate and rhodamin 640 , which quantum efficiency does not depend on the photon energy.

\subsection{The first-principles studies}

The theoretical studies were performed using the plane-wave pseudopotential method [16] implemented in CASTEP package [17]. The optimized norm-conserving pseudopotential [18] in the KleinmanBylander form [19] for carbon in the cubic diamond and lonsdaleite is used to ensure a small plane-wave basis set without compromising the accuracy required by our study. A very high kinetic energy cutoffs of $800 \mathrm{eV}$ for those crystals are used. Monkhorst-Pack $k$-point meshes [20] with the spanning of less than $0.04 / \AA^{3}$ in the Brillouin zone are chosen. For the Raman spectra calculations, the $k$ point mesh density is chosen as high as $0.02 / \AA^{3}$. The convergence testing showed that the choice 


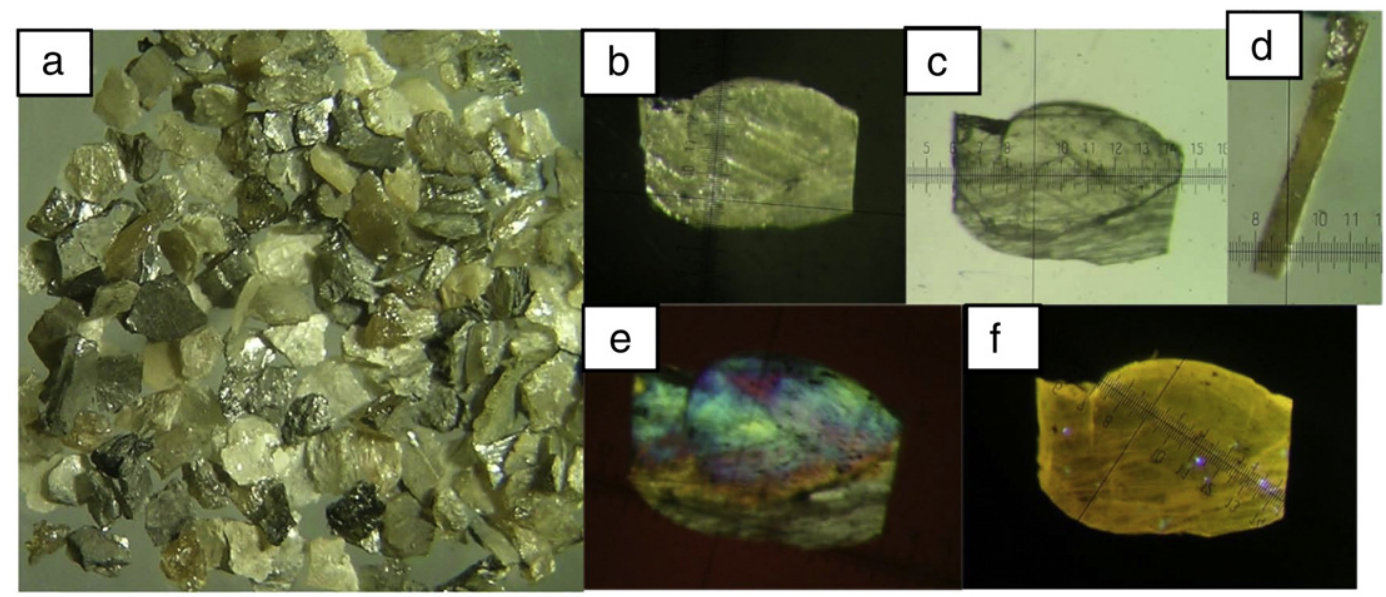

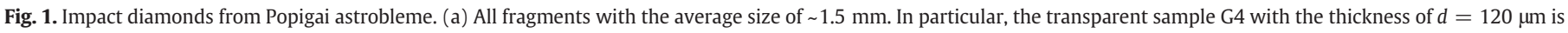
shown at side illumination (b) and in transmitted unpolarized light (c, d). Pattern recorded with crossed polarizers (e) and PL at 365 nm Hg excitation (f).

of the computational parameters is sufficiently accurate for present purposes.

It is known that the density functional theory (DFT) cannot describe the electronic excitations accurately since the exchange-correlation (XC) potential is discontinuity as an electron is added with a given momentum to the system. Indeed, the local density approximation (LDA) or generalized gradient approximation (GGA) of the XC functional is usually underestimating the energy band gap of crystals. In recent years, a wide variety of nonlocal functionals have been developed. In particular, the screened exchange plus LDA correlation (sX-LDA) [21] has been successfully applied in the prediction of the energy band gap of semiconductors. Our tests reveal that the calculated bulk lattice parameters of lonsdaleite and cubic diamond with different XC functionals are all in good agreement with the experimental results (see Table S1 in the Supplementary data). In this work, the energy band gap with the XC functionals of GGA, LDA and sX-LDA for the cubic diamond and lonsdaleite are theoretically determined, respectively, and compared with the experimental values.

Based on the electronic structures, the optical absorption and transmission spectra can be calculated from the electronic transition between the occupied and unoccupied states caused by the interaction with photons [22]:

$\varepsilon_{2}(\hbar \omega)=\frac{2 e^{2} \pi}{\Omega \varepsilon_{0}} \sum_{k, v, c}\left|\left\langle\psi_{k}^{c}|\hat{u} \cdot-r| \psi_{k}^{v}\right\rangle\right|^{2} \delta\left(E_{k}^{c}-E_{k}^{v}-\hbar \omega\right)$

where $\Omega$ is the volume of the elementary cell, $v$ and $c$ represent the valence and conduction bands, respectively, $\omega$ is the frequency of the incident light, and $\hat{u}$ is the vector defining the polarization of the electric field of the incident light. For the GGA and LDA calculations, the scissors operator [23] is introduced to shift all the conduction bands in order to agree with the measured value of the band gap.

The phonons (or lattice vibrations) of the cubic diamond and lonsdaleite are determined by the linear response calculations [24], from which the dynamical matrices (or Hessians) and Born effective charges can be obtained. The Born effective charge tensor of an ion is the partial derivative of the macroscopic polarization with respect to a periodic displacement of all the periodic images of that ion at zero macroscopic electric field [24]. Once these data are given, the Raman spectra are generated by calculating the polarization for each displacement in the linear response frame [25]. It should be noted that in experiments the intensity of peaks in Raman spectra also strongly depends on some other factors such as the temperature and incident light wavelength. These parameters must be specified in order to match the calculated and experimental results well.

\section{Results and discussion}

\subsection{Transmission spectra in UV and near-IR region}

Transmission spectra of four impact diamonds G1, G2, G4 and G6 from Popigai crater are shown in Fig. 2(a). Samples transmit the light from the photon energy of $4.96 \mathrm{eV}$ (wavelength $\sim 250 \mathrm{~nm}$ ), but the signal increases very slowly as shown by curves $1-4$, which is similar to that in CVD diamond film (curve 5) given for comparison. On the contrary, type Ila high quality sample of cubic diamond demonstrates a fast increase of transmission beginning from $5.51 \mathrm{eV}$ $(\sim 225 \mathrm{~nm}$ ) (curve 6). Slow transmission increase in impact diamonds and CVD films is a result of strong Rayleigh scattering on crystalline and phase boundaries as well as on other extended defects (e.g., cracks and dislocations) present in the samples.

In such strongly scattering light, it is very difficult to arrange a precise analysis of the shape of the spectrum near the fundamental absorption edge and to indicate the band gap value and the type of electronic band-to-band transitions. The shape of the spectra in the UV to near-IR does not change even the samples is cooled to $80 \mathrm{~K}$, and no fine structure appears. Therefore, the energy band gap $\left(E_{g}\right)$ can be roughly estimated from the point when light begins to transmit through the sample. On the $2.5 \%$ transmission level the optical absorption edge of the impact diamonds G1, G2, G4 and G6 are located at $4.12,4.67,4.39$ and $4.96 \mathrm{eV}$, respectively (shown in the insert of Fig. 2(a)). Thus, the energy band gaps of impact diamonds are about 0.5 to $1 \mathrm{eV}$ smaller than that of cubic diamonds where $E_{\mathrm{g}}=5.46 \mathrm{eV}$ at $300 \mathrm{~K}[13,26]$.

It is known that in carbon based compounds which are transparent in the visible region the transmission at short wavelengths is determined by the phases with $s p^{3}$ hybridization, namely cubic diamond and lonsdaleite [27]. In addition, the SiC phase which has been identified in impact diamonds by structural analysis may have some contribution [5]. Carbon phases with $s p^{1}$ and $s p^{2}$ hybridization such as graphite, graphene, chaoite, and carbines are characterized by dark (black) color as well as much higher electrical conductivity. These phases are present as thin layers or inclusions in some fragments/samples.

In order to elucidate the UV to near-IR transmission spectra of impact diamond, the first-principles simulations were performed. There is some difference in band gap values obtained by the LDA, GGA and sX-LDA calculations: values by the latter approximation are considerably higher and they are in good agreement with the experimental values (see Table S1 in the Supplementary data). The sX-LDA calculated transmission spectra for lonsdaleite and cubic diamond are displayed in Fig. 2(b). Spectrum of cubic diamond matches the experimental result 


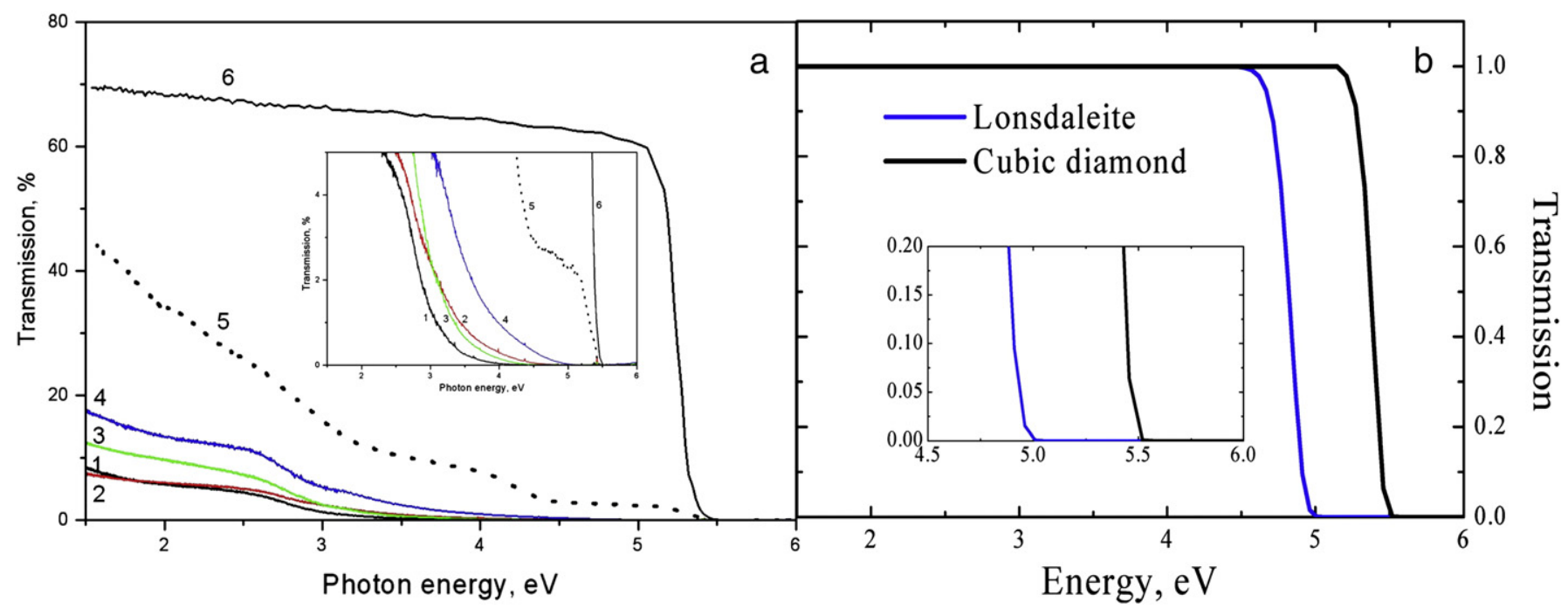

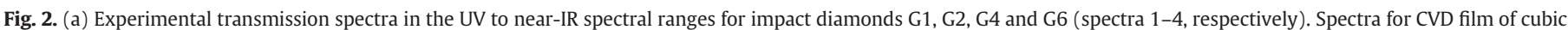

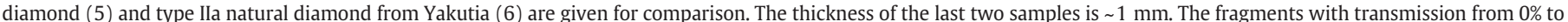
$5 \%$ are shown in the insert. (b) Calculated transmission spectra for cubic diamond and lonsdaleite. The spectra closed to the UV absorption edge are shown in the insert.

for type Ila high quality sample of cubic diamond (curve 6 in Fig. 2(a)) very well. For lonsdaleite, the transparency edge is red-shifted about $0.5 \mathrm{eV}$.

The sX-LDA energy band structures (see Fig. S2 in the Supplementary data) demonstrate that both lonsdaleite and cubic diamond are indirect band gap semiconductors. The direct electronic transition is realized at $\Gamma$ point at $6.25 \mathrm{eV}$ and $7.12 \mathrm{eV}$ for lonsdaleite and cubic diamond, respectively. The latter value is close to the experimental value of $7.3 \mathrm{eV}$ [13]. The band gap is indirect because the wave vector at which the valence band is a maximum $(G)$ does not coincide with the wave vector where the conduction band is a minimum at $\mathrm{L}$ point for cubic diamond and at $\mathrm{K}$ point for hexagonal one. The detailed analysis of the $2 \mathrm{~s}$ and $2 p$ orbitals in the electronic structure of both crystals shows that the width of the valence band is $22 \mathrm{eV}$ in both cases (see Fig. S3 in the Supplementary Data), which is also in good agreement with the experimental value of $20 \mathrm{eV}$ [13]. It should also be noted that in cubic and hexagonal diamonds the shape of the $2 s$ orbitals is very similar to each other, while their $2 p$ orbitals are some different. The difference is mainly due to the crystal structural features of cubic diamond and lonsdaleite. Although both structures have covalent tetrahedral bonds and contain only six-membered rings of bonds, the stacking manner of the (111) atomic planes is different. The staking sequence in lonsdaleite is of the type of $A B C A B C$... (each atomic layer designated by $A, B$, or $C$ ), whereas that in cubic diamond is of the type of $A B A B A B \ldots .$. The lengths of some carbon-carbon bonds in lonsdaleite are slightly larger ( $1.5 \%$ larger) than those in cubic diamond [28], which results in the smaller energy band gap in the former crystal. Calculated band gaps are 5.34 and $4.55 \mathrm{eV}$ for cubic and hexagonal diamonds whereas experimental values are 5.5 and $4.96 \mathrm{eV}$, respectively. Calculated value for lonsdaleite is similar to that obtained in Ref. [29], but differs considerably from $3.5 \mathrm{eV}$ in Ref. [30].

Based on the above analysis, one may conclude that the band gap modifications in the impact diamonds are mainly due to the lonsdaleite input, consistent with the studies by Bakr et al. [31]. On the other hand, it is difficult to explain the band gap narrowing in the samples by the presence of $\mathrm{SiC}$ layers because different $\mathrm{SiC}$ phases have considerably lower $E_{g}$ values: they are 2.36, 3.23 and $3.05 \mathrm{eV}$ for $3 \mathrm{C}(\beta), 4 \mathrm{H}$ and $6 \mathrm{H}(\alpha)$ phases, respectively $[32,33]$. Thus, absorption edge for these phases should be located near 525, 383 and $406 \mathrm{~nm}$ respectively, far away from the measured absorption edge.

\subsection{Mid-IR absorption spectra}

In Fig. 3, the mid-IR absorption spectra for four impact diamond samples are shown in section (a) in comparison with cubic diamonds of different types in frameworks of well-known physical classification (types Ib, IaA and IaAB in section (b) $[8,13]$ ). In spectra of impact diamonds the absorption bands at 2000 and $2160 \mathrm{~cm}^{-1}$ are related to the multiphonon absorption of cubic diamond lattices, although their structures are somewhat less pronounced. The absorption at $v=1995 \mathrm{~cm}^{-1}$ in $3 \mathrm{C}$ bulk diamonds has been reliably determined to be $12.3 \mathrm{~cm}^{-1}$ and many authors use it as an internal standard [13]. To date, there is no reliable information concerning absorption spectrum of lonsdaleite. Taking into account the presence of both cubic and hexagonal phases in impact diamonds, it was interesting to measure the absorption at $v=1995 \mathrm{~cm}^{-1}$. The results of such measurements for 4 flattened samples G1, G2, G4, and G6 are given in Table 1. One can see that at this frequency the absorption is approximately the same for G1 and G2 and a little higher for G4 $\left(15 \mathrm{~cm}^{-1}\right)$. Somewhat lower absorption for G6 may be a result of its wedge-like shape (See Table S2 in the Supplementary data). One can conclude that lattice absorption in the $1800-2500 \mathrm{~cm}^{-1}$ is approximately the same for cubic and hexagonal diamonds. Some deviations may be due to the nanocrystalline structure.

The unusual absorption in the one-phonon region $\left(v<1400 \mathrm{~cm}^{-1}\right)$ in impact diamonds may be associated with the native structural defects or impurities in the lonsdaleite phase.

The shape of the spectrum varies from point to point or from sample to sample but components remain the same: Typically they are at $969,1102,1225$ and $1330 \mathrm{~cm}^{-1}$. They are shown by dotted lines in Fig. 3(a). In particular, the absorption spectrum (1) of impact diamond with a maximum at $1225 \mathrm{~cm}^{-1}$ is very similar to that of low-nitrogen nano-polycrystalline $3 \mathrm{C}$ diamond synthesized directly from graphite under high pressure and temperature [34]. These bands have no analogs to the spectra of the cubic diamonds in one-phonon region which are determine mainly by nitrogen defects; a single nitrogen atom in carbon site (center $\mathrm{C}$, with dominating band at $1135 \mathrm{~cm}^{-1}$ ) for type Ib diamonds, two nitrogen atoms in adjacent sites (center $\mathrm{A}$, at $1280 \mathrm{~cm}^{-1}$ ) for type IaA and nitrogen-vacancy complexes $\mathrm{N}_{4} \mathrm{~V}$ (center $\mathrm{B}$, at $1175 \mathrm{~cm}^{-1}$ ) for type IaB. This means that the impact diamond samples do not contain the mentioned 


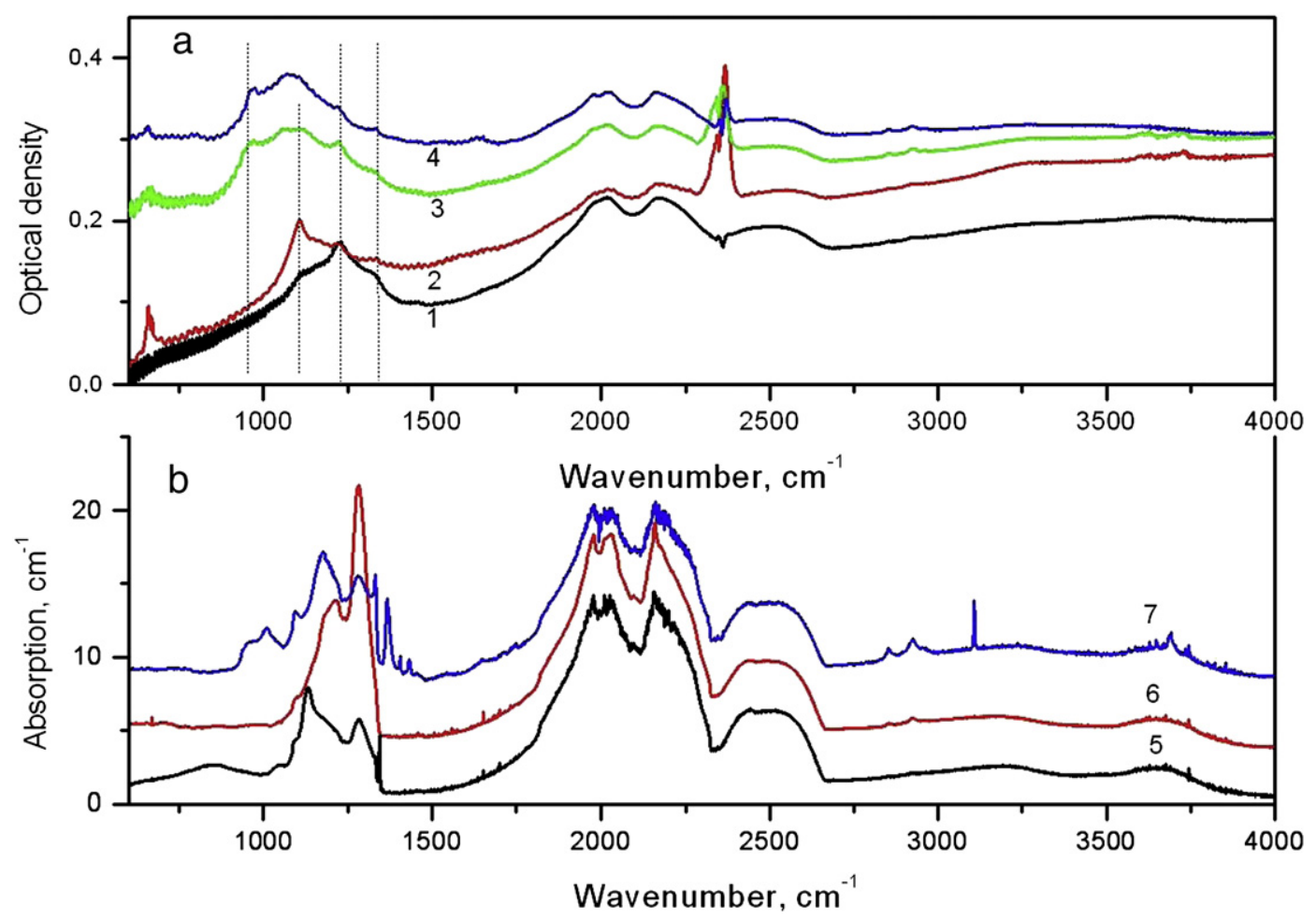

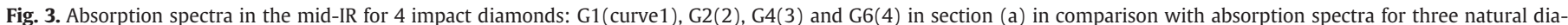
monds (b) of types Ib (5), IaA (6) and IaAB (7). Spectra 2-4 and 6-7 are shifted upwards for clarity. Here, optical density is D = lg (100 / I).

nitrogen impurities in concentration more than $10^{17} \mathrm{~cm}^{-3}$. Narrow lines in the $1358-1372 \mathrm{~cm}^{-1}$ range in natural diamonds are due to $\mathrm{B}^{\prime}$ carbon aggregates or platelets (curve 7 in Fig. 3(b)) $[8,13]$.

In impact diamonds there are two groups of lines in the ranges from 658 to $645 \mathrm{~cm}^{-1}$ and from 2406 to $2362 \mathrm{~cm}^{-1}$ which have been previously interpreted as the $\nu_{3}$ and $\nu_{2}$ bands of $\mathrm{CO}_{2}$ due to inclusions of pressurized solid carbon dioxide $\left(\mathrm{CO}_{2}\right)$ in cubic diamonds [13]. This feature is typical of natural type IaA diamonds as powder with grain size below $250 \mathrm{~nm}$ after annealing at $700{ }^{\circ} \mathrm{C}$ in an oxygen atmosphere [13]. The narrow line at $3107 \mathrm{~cm}^{-1}$ results from the $\mathrm{C}-\mathrm{H}$ vibrations.

A periodic structure in the 600 to $1100 \mathrm{~cm}^{-1}$ (10 to $\left.15 \mu \mathrm{m}\right) \mathrm{spec}-$ tral range in the mid-IR (see Fig. S4 in the Supplementary Data) is a result of interference between two flat and parallel faces. The analysis of extremum position following Ref. [31] gives the plate thickness which is in good agreement with values measured directly (under an optical microscope or with a micrometer). Thus transparent plates of impact diamond behave themselves in the mid-IR as if there is a

Table 1

Spectroscopic features in mid-IR absorption spectra of impact diamonds.

\begin{tabular}{|c|c|c|c|}
\hline $\begin{array}{l}\text { Sample } \\
\text { NN }\end{array}$ & $\begin{array}{l}\text { Absorption at } \\
1995 \mathrm{~cm}^{-1}\end{array}$ & $\begin{array}{l}\mathrm{CO}_{2} \text { absorption } \\
\left(657,2380 \mathrm{~cm}^{-1}\right)\end{array}$ & $\begin{array}{l}\text { Absorption bands in one-phonon } \\
\text { spectral range, } \mathrm{cm}^{-1}\end{array}$ \\
\hline \multicolumn{4}{|c|}{ Impact diamonds from Popigai crater } \\
\hline G1 & $12.7 \pm 0.5$ & + & $1102,1225,1330$ \\
\hline G2 & $15.0 \pm 1.0$ & + & $\overline{969}, 1102,1225,1330$ \\
\hline G4 & $6.9 \pm 3$ & + & $\overline{969}, \overline{110,} 1 \overline{225,} 1330$ \\
\hline G6 & $12.3 \pm 0.5$ & - & $\overline{110} 2, \underline{1225}, 1330$ \\
\hline \multicolumn{4}{|c|}{ Natural kimberlite (cubic) diamonds } \\
\hline Type Ib & 12.3 & & 1135 - center C \\
\hline $\begin{array}{l}\text { Type } \\
\text { IaA }\end{array}$ & 12.3 & & 1282 - center A \\
\hline $\begin{array}{l}\text { Type } \\
\text { IaAB }\end{array}$ & 12.3 & & 1175,1282 - centers $B^{\prime}$ and $A$ \\
\hline
\end{tabular}

Most intense peaks are underlined. relatively homogeneous, monolithic substance without any substructure inside.

\subsection{Raman spectra}

Raman spectroscopy is commonly used in everyday practice for the characterization and identification of the various phases of carbon in diamond films and other structures. The Raman spectra of various carbon phases are well known [35]. The first-order Raman spectrum of cubic diamond consists of a single narrow Lorentzian-shaped peak at $1332.5 \mathrm{~cm}^{-1}\left(4 \times 10^{13} \mathrm{~Hz}, 165 \mathrm{meV}\right)$, with FWHM of $1.5 \mathrm{~cm}^{-1}$ in an undisturbed diamond lattice. This line results from the scattering on triply degenerate $\mathrm{TO}(\mathrm{X})$ phonons of $\mathrm{T}_{2 \mathrm{~g}}$ symmetry $[36,37,13]$.

Several groups studied experimentally the Raman spectra of diamond and lonsdaleite $\mathrm{sp}^{3}$ aggregates [38-42]. Most of them observed only a broad enough asymmetric line centered near $1332 \mathrm{~cm}^{-1}$ and associated it with usual $\mathrm{T}_{2 \mathrm{~g}}$ vibration in cubic diamond $3 \mathrm{C}$ phase combined with split, but poorly resolved $\mathrm{T}_{2 \mathrm{~g}}$ vibration in lonsdaleite [39-41]. Some authors investigated pure lonsdaleite phase: small single crystals of natural lonsdaleite from the Kokchetav massif in Kazakhstan [42] and a purely lonsdaleite film produced in the hot filament CVD process [43]. The Raman spectra showed the well resolved peaks at 1319 and $1322 \mathrm{~cm}^{-1}$ in the former [42], whereas an unresolved asymmetric line $1329 \mathrm{~cm}^{-1}$ with a shoulder at lower energies were observed in the latter [43]. This implies that the lonsdaleite signal is a doublet in the $1315-1329 \mathrm{~cm}^{-1}$ spectral range, and the variations in line positions are due to a shift to lower energies as temperature/laser beam increase or shift to higher energies as stress grows.

In this work, the Raman measurements are performed on two types of impact diamonds: transparent, slightly colored samples and opaque samples, gray to black in color. They are given in sections (a) and (b) of Fig. 4, respectively. Fig. 4(a) clearly shows that for a transparent sample PID10 there are a set of peaks besides the main 
diamond peak near $1332 \mathrm{~cm}^{-1}$. The results of spectrum decomposition into 8 Lorentzian-shaped components are given in Table 2.

The insert in Fig. 4(a) shows that there is an asymmetric relatively narrow peak near $1332 \mathrm{~cm}^{-1}$ with FWHM $\sim 15 \mathrm{~cm}^{-1}$ for transparent PID samples. This peak consists of at least three components at 1323.5, 1331.6 and $1336 \mathrm{~cm}^{-1}$ with FWHM of 4.7, 5.8 and $7.3 \mathrm{~cm}^{-1}$, respectively. Estimation using the coefficients $\delta v_{[100]}=0.73 \mathrm{~cm}^{-1} / \mathrm{GPa}$ and $\delta v_{[111]}=2.2 \mathrm{~cm}^{-1} / \mathrm{GPa}$ [44] gives the residual stress of 2 to $5 \mathrm{GPa}$ for diamond. Broadening of the components may be a result of samples nanocrystalline structure [34]. The latter has been observed directly at the electron microscopy examination [5].

The intense peak at $1082 \mathrm{~cm}^{-1}$ is recorded only at $514.5 \mathrm{~nm}$ excitation and is absent at $325 \mathrm{~nm}$ excitation; it correlates in intensity with PL luminescence and can be interpreted as a zero-phonon line (ZPL) at $544.8 \mathrm{~nm}$. Indeed, several ZPLs, at $2.23 \mathrm{eV}(556 \mathrm{~nm})$, $2.32 \mathrm{eV}(534 \mathrm{~nm})$ and $2.39 \mathrm{eV}(519 \mathrm{~nm})$ were observed in the 500 to $550 \mathrm{~nm}$ range in the low-temperature CL spectra of impact diamonds from the Popigai, Ries and Lappajarvi craters [12]. At room temperature the ZPL positions can be different.

The $1180 \mathrm{~cm}^{-1}$ component is originated from the phonon on the edge of Brillouin zone [37], whereas the $1460 \mathrm{~cm}^{-1}$ component is associated with the $\mathrm{sp}^{2}$ amorphous-type graphitic bonding [38]. An alternative interpretation is that the 1180 and $1460 \mathrm{~cm}^{-1}$ bands are the $v_{1}$ and $v_{3}$ components of trans-polyacetylene, respectively [35]. The broad bands near 500 and $750 \mathrm{~cm}^{-1}$ are observed in nanocrystalline diamonds as well but their origin is not clear to date.

The detailed Raman spectra of the transparent PID samples in the low-energy region $\left(v<800 \mathrm{~cm}^{-1}\right.$, see Fig. S5) exhibit the structural characteristics of impurities: they are related to non-carbon phases such as quartz $\mathrm{SiO}_{2}(1)$, magnetite $\mathrm{Fe}_{3} \mathrm{O}_{4}$ (2) and hematite $\alpha-\mathrm{Fe}_{2} \mathrm{O}_{3}$ (3). These spectra were identified with the help of Databank [45] (the positions of the components see Table S3 in the Supplementary data). It should be noted that specific peaks are shifted to high energies compared with the value in Ref. [45], which is a result of residual stresses of minerals. Thus fundamental vibrations in quartz, magnetite and hematite are shifted 5,25 and $17 \mathrm{~cm}^{-1}$, respectively. Taking
Table 2

The results of decomposition of the Raman spectrum for transparent IDP sample into 8 individual Lorentzian components (at $514.5 \mathrm{~nm}$ excitation).

\begin{tabular}{|c|c|c|c|}
\hline NN & $\begin{array}{l}\text { Position of the peak } \\
\text { maximum }\left(\mathrm{cm}^{-1}\right)\end{array}$ & $\begin{array}{l}\text { FWHM } \\
\left(\mathrm{cm}^{-1}\right)\end{array}$ & Interpretation \\
\hline 1 & 550 & 380 & - \\
\hline 2 & 750 & 197 & - \\
\hline 3 & 1082 & 90 & $\begin{array}{l}\text { ZPL at } 544.8 \mathrm{~nm} \text { in the PL } \\
\text { spectrum }\end{array}$ \\
\hline 4 & 1185 & 200 & $\begin{array}{l}\text { Phonon on the edge of Brillouin } \\
\text { zone [37] }\end{array}$ \\
\hline 5 & 1323.5 & 4.7 & Lonsdaleite \\
\hline 6 & 1331.6 & 5.8 & Cubic diamond \\
\hline 7 & 1336 & 7.3 & Cubic diamond \\
\hline 8 & 1460 & 160 & $\begin{array}{l}\mathrm{sp}^{2} \text { amorphous-type graphitic } \\
\text { bonding [38] }\end{array}$ \\
\hline
\end{tabular}

into account that fundamental vibration at $463 \mathrm{~cm}^{-1}$ shifts at the rate $\delta v=9 \mathrm{~cm}^{-1} / \mathrm{GPa}$ as pressure increases [46] we obtain that the estimated residual stress is $\sim 0.6 \mathrm{GPa}$ in quartz. For magnetite and hematite, the dependence of Raman-active fundamental vibrations on pressure is considerably nonlinear and estimated residual pressures are 10 and $20 \mathrm{GPa}$, respectively [47,48].

The Raman spectra of the gray or black impact diamonds (PID 1-3) in Fig. 4(b) exhibit two main peaks at $\sim 1585$ and $1355 \mathrm{~cm}^{-1}$. The peak at $1585 \mathrm{~cm}^{-1}$ is originated from the first-order spectrum of the $E_{2 g}$ optical mode ( $\mathrm{G}$ mode) in well-ordered defect-free crystalline graphite at $1568.5 \mathrm{~cm}^{-1}$ which is shifted due to the graphite disorder [49]. Meanwhile, the wide FWHM of the G line indicates the presence of glassy graphite $\left(60-80 \mathrm{~cm}^{-1}\right)$ and pyrolitic graphite $\left(23 \mathrm{~cm}^{-1}\right)$ in the samples since this parameter is $\sim 13 \mathrm{~cm}^{-1}$ for natural graphite crystals. The line near $1355 \mathrm{~cm}^{-1}$ (D mode) was first reported by Tuinstra and Koenig [50] and corresponds to a disorder-induced mode. It is known that the ratio of the intensities for D and G bands depends on the size of the graphite microcrystals in sample [50]. Accordingly, we calculated the ratio of $\mathrm{I}(1355) / \mathrm{I}(1585)$ and estimated that the crystallite sizes in

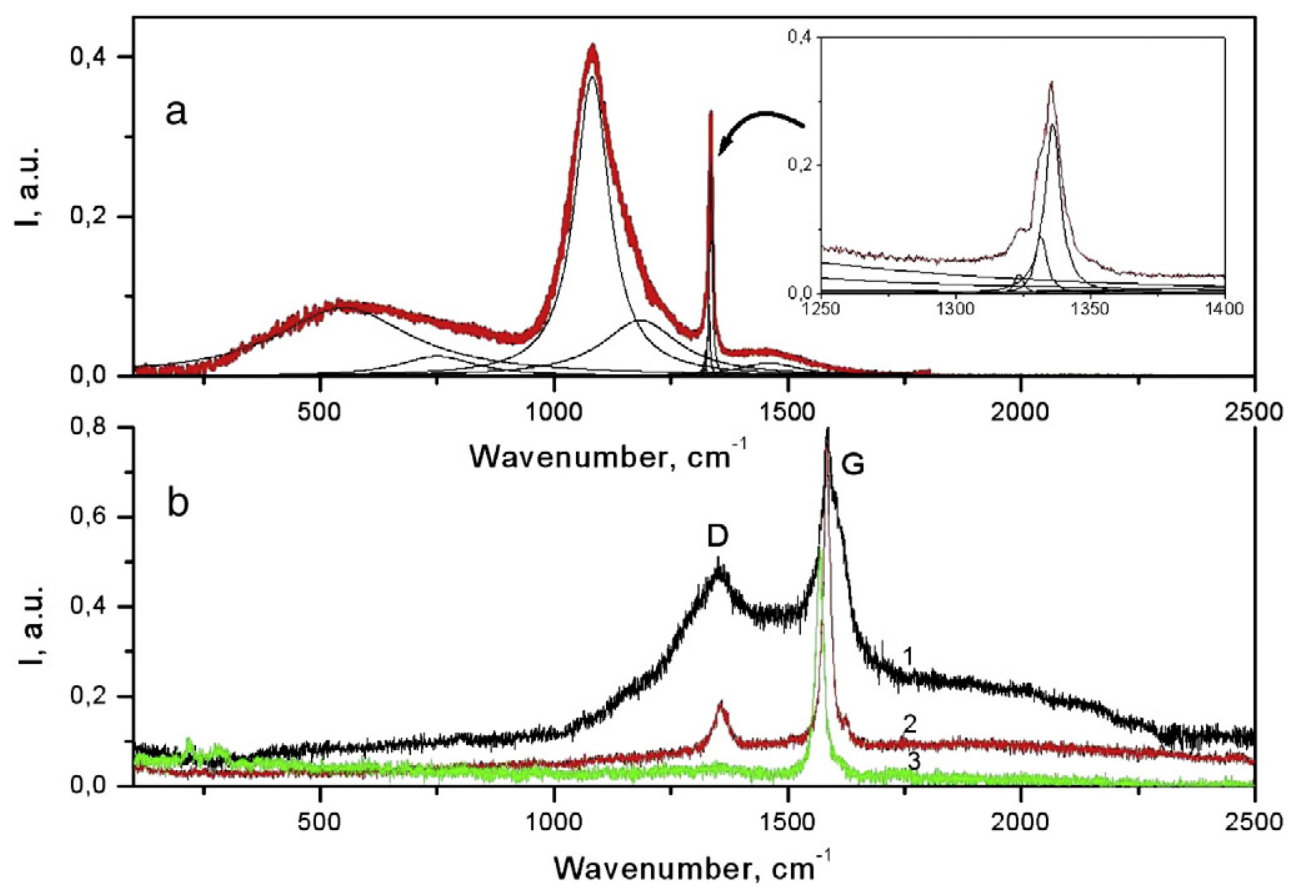

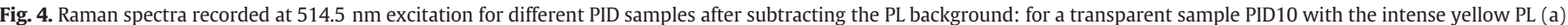

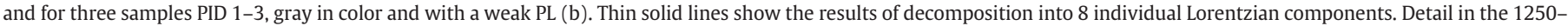

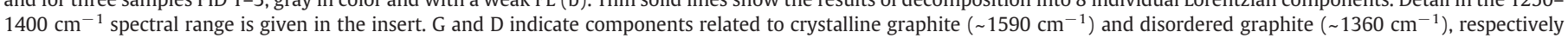
[36]. 
Table 3

Calculated frequencies and intensity of the IR peaks for cubic diamond and lonsdaleite. The symbols S, D, T represent single (non-degenerate), double and triple degenerate modes, respectively.

\begin{tabular}{|c|c|c|c|c|c|}
\hline & Frequency & Intensity & Frequency & Intensity & Selection rules \\
\hline $\begin{array}{l}\text { Cubic } \\
\text { diamond }\end{array}$ & This work & & $\begin{array}{l}\text { Experimental } \\
{[35]}\end{array}$ & & \\
\hline $\mathrm{T}_{1 \mathrm{~g}}(\mathrm{~T})$ & 0 & & & & \\
\hline $\mathrm{T}_{2 \mathrm{~g}}(\mathrm{~T})$ & 1315 & 1 & 1332 & & Raman (xz, yz, xy) \\
\hline Lonsdaleite & This work & & $\begin{array}{l}\text { Other work } \\
\text { [41] }\end{array}$ & & \\
\hline $\mathrm{E}_{1 \mathrm{u}}(\mathrm{D})$ & 0 & & - & & - \\
\hline $\mathrm{A}_{2 \mathrm{u}}(\mathrm{S})$ & 0 & & - & & - \\
\hline $\mathrm{E}_{2 \mathrm{u}}(\mathrm{D})$ & 529 & & 524 & & Inactive \\
\hline $\mathrm{B}_{1 \mathrm{u}}(\mathrm{S})$ & 1065 & & 1055 & & Inactive \\
\hline$E_{2 g}(D)$ & 1206 & 0.26 & 1221 & 0.1 & $\operatorname{Raman}\left(x^{2}-y^{2}, x y\right)$ \\
\hline$B_{2 g}(S)$ & 1252 & & 1252 & & Inactive \\
\hline$A_{1 g}(S)$ & 1285 & 1 & 1280 & 1 & $\operatorname{Raman}\left(\mathrm{x}^{2}+\mathrm{y}^{2}, \mathrm{z}^{2}\right)$ \\
\hline$E_{1 g}(D)$ & 1319 & 0.18 & 1338 & 0.1 & Raman (yz, xz) \\
\hline
\end{tabular}

the samples PID 1-3 are $10 \mathrm{~nm}, 20 \mathrm{~nm}$ and $100 \mathrm{~nm}$, respectively (see Table S4 in the Supplementary data).

In order to elucidate the origins of the Raman spectra for the cubic diamond and lonsdaleite, the first-principles studies are performed and the results are listed in Table 3 and plotted in Fig. 5(a). Since the strong ZPL bands in our samples may affect the exhibition of the refined spectrum structures in the $s p^{3}$ carbon phases, the experimental Raman spectrum for the diamond-lonsdaleite system by the HPHT process [41] is adopted to compare with the calculated results. Clearly, their perfect agreement is achieved, not only for the peak positions, but also for their intensity ratio, after aligning the calculated Raman active peak of cubic diamond with the experimental position by entirely shifting the calculated spectra upward to the higher energy by $17 \mathrm{~cm}^{-1}(\sim 2 \mathrm{meV})$. The very small discrepancy might be due to the difference between the simulated and experimental conditions such as temperature and stress present in samples. In all optical models only the $A_{1 g}, E_{1 g}$ and $E_{2 g}$ modes at 1302, 1336 and $1223 \mathrm{~cm}^{-1}$ (after correction), respectively, are Raman active in lonsdaleite (see Table 3).

Fig. 5(b) displays the atomic vibrational modes with Raman activity for cubic diamond and lonsdaleite. The $\mathrm{A}_{\mathrm{lg}}$ and $\mathrm{E}_{1 \mathrm{~g}}$ modes for lonsdaleite, correspond to the triply-degenerate stretching mode of cubic diamond $\mathrm{T}_{2 \mathrm{~g}}$ which splits into a component vibrating perpendicular to and a component in the plane of the hexagonal layers, respectively. Unlike graphite, the bonding strength of hexagonal diamond along these two directions is comparable, so the wavenumber separation between the $A_{\lg }$ and $E_{1 g}$ modes is expected to be small. In analogy to wurtzite, the third Raman band of $E_{2 g}$ symmetry is a shear mode and occurs in the low wavenumber region. Like cubic diamond, the hexagonal diamond structure has no first order infrared absorption.

\subsection{Photoluminescence spectra}

Fig. 6 exhibits the typical low-temperature photoluminescence spectra of impact diamonds at 4.6 and $6.9 \mathrm{eV}$ excitations using synchrotron radiation. Both PL spectra can be decomposed as at least three individual components (their positions and FWHM see Table S5 in the Supplementary data). The low energy components at 2.06 and $2.38 \mathrm{eV}$ are dominating at $4.6 \mathrm{eV}$ excitation, whereas a high energy component at $2.84 \mathrm{eV}$ is more intense at $6.9 \mathrm{eV}$ excitation (Fig. 6). As a result the PL emission is bluish-white at $6.9 \mathrm{eV}$ excitation and white-yellow at $4.6 \mathrm{eV}$ excitation. Similar broad bands near 1.8 and $2.8 \mathrm{eV}$ were observed as well in CL spectra of impact diamonds at 80 and $300 \mathrm{~K}$ [12].

A specific feature of the PL spectra of impact diamonds including those from Popigai astrobleme is the broad band emission and excitation spectra with FWHM $\sim 100 \mathrm{~nm}(0.4 \mathrm{eV})$ whereas vibronic systems with the well-pronounced narrow ZPLs are typical for both natural kimberlite and synthetic cubic diamonds. These PL bands are associated with the well-known vibronic systems N3, H3 and 575 for cubic diamonds [13]. These systems are due to nitrogen-vacancy complexes $\mathrm{N}_{3} \mathrm{~V}, \mathrm{VN}_{2} \mathrm{~V}$ and $\mathrm{NV}^{0}$, where $\mathrm{N}$ is a nitrogen atom and $\mathrm{V}$ is vacancy. The ZPLs are located at $2.985 \mathrm{eV}$ (415.2 nm), $2.463 \mathrm{eV}$ (503.2) and $2.156 \mathrm{eV}(575 \mathrm{~nm})$ whereas broad bands maximums are at 2.81, 2.6 and $2.05 \mathrm{eV}$, respectively [13]. Absence of fine structure in examined diamonds is supposed to be a result of (i) nanocrystallized structure of impact diamonds and (ii) strong deformations in the lattice at solid state phase transition. Parameters of the shock process during the Popigai event were estimated to be the following: pressure of $\sim 35$ to $50 \mathrm{GPa}$, temperature up to $3000{ }^{\circ} \mathrm{C}$ and process duration of $\sim 1 \mathrm{~s}$ [9]. On the other hand broad band PL without any fine structure in the 550 to $800 \mathrm{~nm}$ range centered at $\sim 2.0 \mathrm{eV}(\sim 620 \mathrm{~nm})$ was observed also in many studies of amorphous hydrogenated carbon material (a-C:H) [51,52], CVD diamond films and polycrystalline diamonds: all

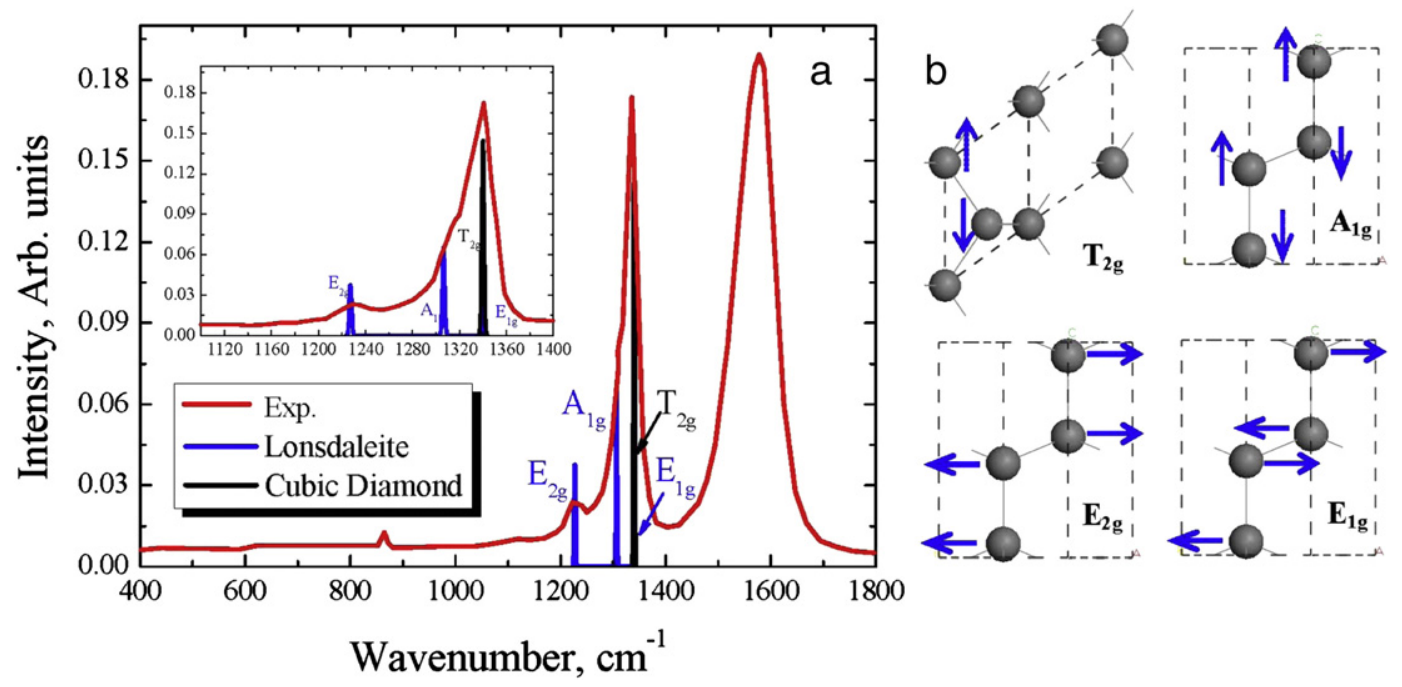

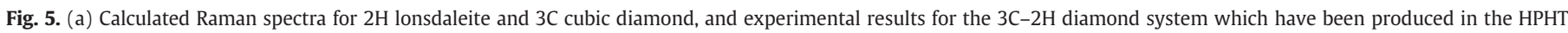

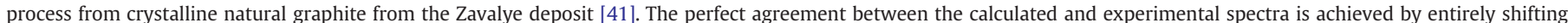

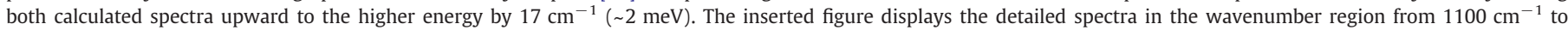

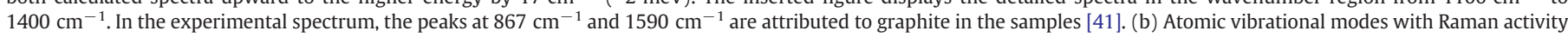
in lonsdaleite and cubic diamonds. 


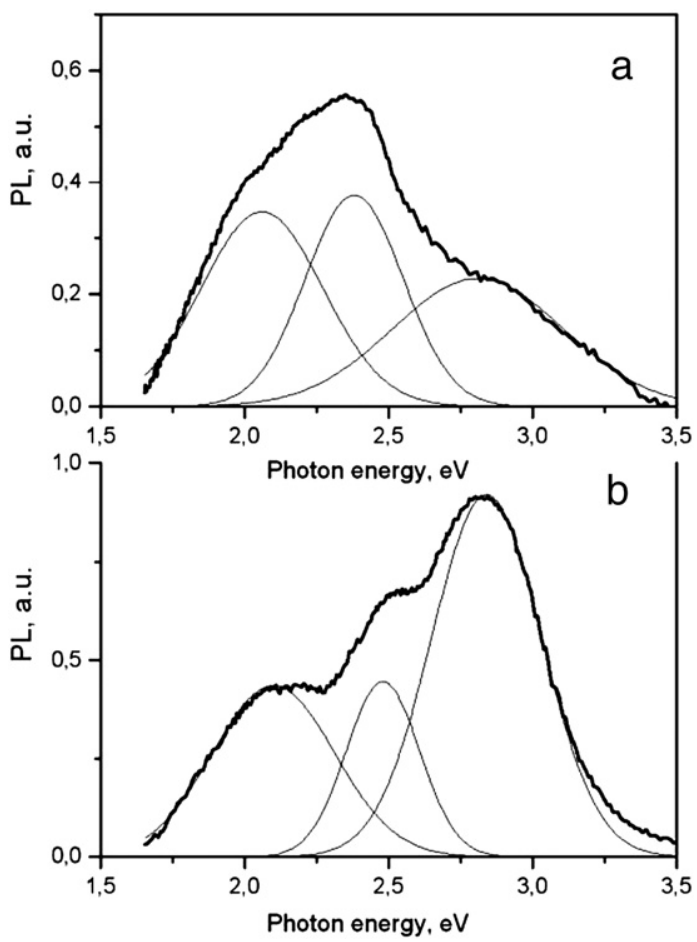

Fig. 6. Photoluminescence spectra of impact diamond from the Popigai astrobleme, recorded at $4.6 \mathrm{eV}(270 \mathrm{~nm})$ excitation (a) and at $6.9 \mathrm{eV}(180 \mathrm{~nm})$ excitation (b). T $=8 \mathrm{~K}$. Spectral resolution is $0.02 \mathrm{eV}(\sim 2 \mathrm{~nm})$. Results of decomposition to Gaussians are shown by thin solid lines. Parameters of Gaussians are given in Table 2.

these materials possess a random network of $s p^{2}$ and $s p^{3}$ bonding configuration [53]. One associates such broadband PL just with $s p^{2}$ amorphous-like carbon bonding in the diamond lattice.

In high resolution PL spectra recorded with a $514.5 \mathrm{~nm}$ excitation we observed sometimes a doublet of narrow lines near $1.7852 \mathrm{eV}$ $(694.3 \mathrm{~nm})$ and $1.78888 \mathrm{eV}(692.9 \mathrm{~nm})$ with $\mathrm{FWHM} \sim 0.5 \mathrm{~nm}$ $(\sim 1 \mathrm{meV})$, both in the strongly luminescent transparent PID samples and gray to black PIDs with weak PL (see Fig. S6 in the Supplementary data). In some transparent PIDs there was also a structured Raman spectrum related to other minerals, whereas in gray PIDs, one could observe only this doublet in PL. We suppose that these two lines are $R_{1}$ and $R_{2}$ lines corresponding to electronic transitions ${ }^{2} E \rightarrow{ }^{4} A_{2}$ in $\mathrm{Cr}^{3+}$ ions. These spin-forbidden transitions are typical of many minerals and their position very weakly depends on crystal field [54]. This moment it is not clear where are these $\mathrm{Cr}^{3+}$ ions located in impact diamonds: most likely they are point defects in mineral inclusions although we cannot exclude a version that they are inside $\mathrm{sp}^{3}$ nanodiamond domains or at least between the grains. We observed also a slightly pronounced structure in the $600-700 \mathrm{~nm}$ range following Ref. [5] and it will be a subject of further investigation.

PL excitation spectra for high energy $2.84 \mathrm{eV}$ component and low energy $2.1 \mathrm{eV}$ components, measured at 2.9 and $2.14 \mathrm{eV}$, respectively are shown in Fig. 7. One can see that blue $2.9 \mathrm{eV}$ emission is excited at photon energies of $\mathrm{h} v>5.0 \mathrm{eV}$ in a broad band centered at $6.25 \mathrm{eV}$. Yellow to orange PL is excited not only in a broad $6.6 \mathrm{eV}$ band but also in two broad bands with maximums at 2.4 and $3.0 \mathrm{eV}$. It is obvious that $\mathrm{h} v>5.0 \mathrm{eV}$ excitation corresponds to band-to-band electronic transitions whereas 2.4 and $3.0 \mathrm{eV}$ bands are due to intracenter transitions from ground to excited state transitions in two different point defects, respectively. These two bands may be related to above mentioned 575 and $\mathrm{H} 3$ centers when their spectra are strongly broadened because of residual stresses. The $8 \mathrm{eV}$ band in the luminescence excitation spectra (Fig. 7) is associated with direct allowed electronic transitions. Decrease of the PL intensity behind the fundamental absorption edge is explained usually by nonradiative recombination on the sample surface. Maximums at 21.7 and $38 \mathrm{eV}$ reflect features of state density distribution in the depth of valence and conduction bands.

\section{Conclusions}

i. Impact diamonds were found to consist of different carbon phases: cubic and hexagonal diamond ( $s p^{3}$-bonding from X-ray structural analysis) as well as amorphous, crystalline and disordered graphite ( $s p^{2}$-bonding, Raman scattering). The sizes of graphite domains vary from 10 to $100 \mathrm{~nm}$.

ii. Fundamental absorption edge for Popigai impact diamonds is shifted $\sim 0.5 \mathrm{eV}$ to lower energies in comparison with kimberlite

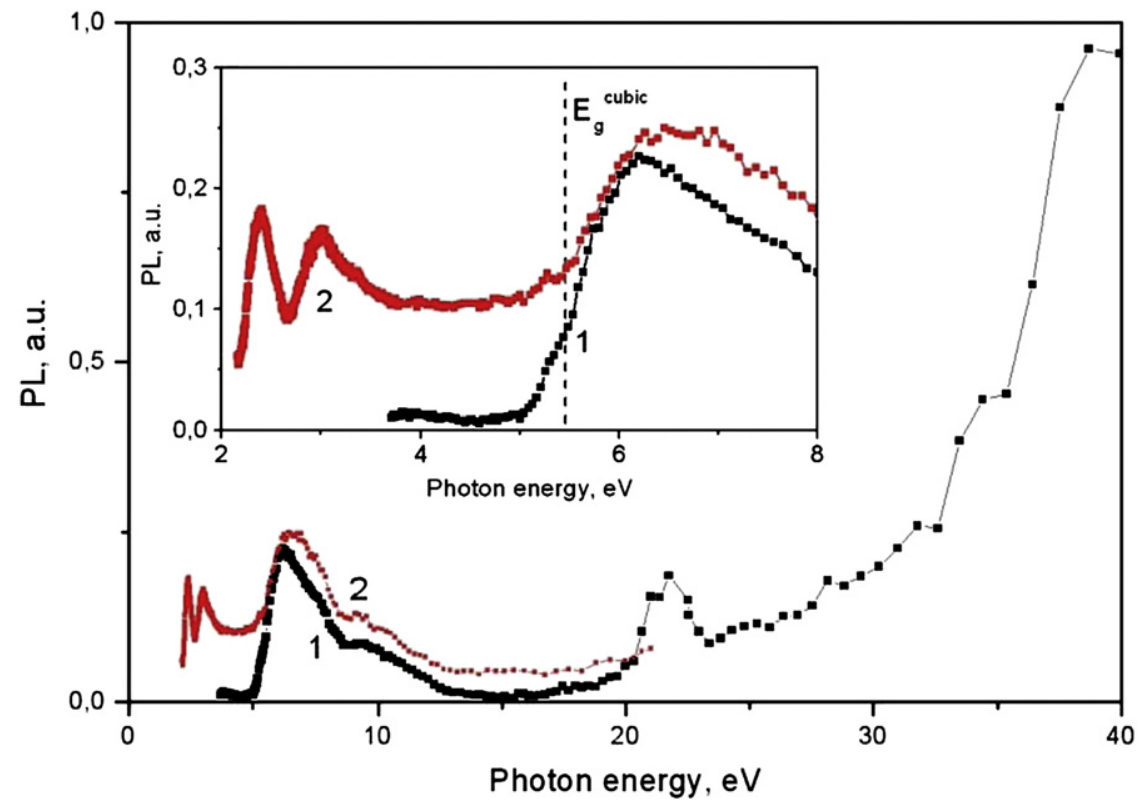

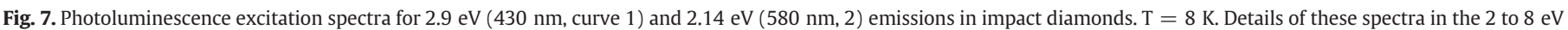
spectral range are given in the insert. Vertical line in the insert shows the band gap for cubic diamond (5.5 eV). 
diamonds ( $5.47 \mathrm{eV}$ ) as a result of the cubic diamond + lonsdaleite structure. Ab initio calculations gives band gap $E_{g}$ values 5.34 and $4.55 \mathrm{eV}$ for cubic $(3 \mathrm{C})$ and hexagonal $(2 \mathrm{H})$ diamonds, respectively. Yellowish color of impact diamonds is likely due to Rayleigh light scattering on structural defects (block and phase boundaries).

iii. Absorption of cubic diamond lattice dominates in the 1800 to $2800 \mathrm{~cm}^{-1}$ range in impact diamonds. Absorption intensity at $1995 \mathrm{~cm}^{-1}$ in impact diamonds is close to $12.3 \mathrm{~cm}^{-1}$, the typical value of cubic diamonds. There is no absorption of nitrogen defects in the one-phonon region in the mid-IR, but there is a set of new bands at $969,1102,1225$, and $1330 \mathrm{~cm}^{-1}$.

iv. Mineral inclusions such as quartz, magnetite, hematite were found in impact diamonds using the Raman spectroscopy. The high energy shift in the peaks position indicates the residual stresses $\sim$ several GPa. Narrow well-resolved $R_{1,2}$ lines in PL spectra indicate the presence of $\mathrm{Cr}^{3+}$ impurity in impact diamonds supposedly in mineral inclusions. The vibration modes from cubic diamond and lonsdaleite were elucidated by the first-principles studies.

v. In most of impact diamonds from the Popigai astrobleme PL spectra are a superposition of broad bands centered at 2.1,2.38, and $2.84 \mathrm{eV}$. All emissions are excited at band-to-band electronic transitions (at $\mathrm{h} v>5 \mathrm{eV}$ ) whereas PL in the low energy component can be excited in 2.4 and $3.0 \mathrm{eV}$ bands. This is the intracenter recombination processes supposedly in the $575(\mathrm{NV})$ and $\mathrm{H} 3$ centers (NVN).

\section{Prime novelty statement}

The optical properties of impact diamonds from the Popigai astrobleme, one of the largest meteorite craters in the world, have been systematically studied. The mechanisms of the absorption, photoluminescence and Raman spectra in the samples are elucidated from the advanced experimental spectroscopy measures combined with the state-of-the-art first-principles theory.

\section{Acknowledgments}

Authors are thankful to I. Kupriyanov and S. Rashchenko for the help in experiments. This work was supported by the Russian Foundation for Basic Research (13-05-00568a), the National Natural Science Foundation of China under Grant Nos. 11174297 and 91022036, and the National Basic Research Project of China (Nos. 2010CB630701 and 2011CB922204).

\section{Appendix A. Supplementary data}

Supplementary data to this article can be found online at http:// dx.doi.org/10.1016/j.diamond.2013.04.008.

\section{References}

[1] H.H. Nininger, Arizona's Meteorite Crater: Its Past, Present and Future, World Press Inc., Denver, Colorado, 1956. 232.

[2] M.V. Yerofeev, P.A. Lachinov, About the Novo Urei meteorite, Zhurnal Russkogo Physiko-Khimicheskogo Obstchestva 20 (1888) 185-213 (in Russian).

[3] A.E. Foote, Am. J. Sci. 42 (1891) 413-417.

[4] G.P. Vdovykin, The Carbon Matter of Meteorites (Organic Compounds, Diamonds, Graphite), Nauka Press, Moscow, 1970. 272, (in Russian).

[5] A.A. Val'ter, G.K. Er'omenko, V.N. Kvasnitsa, Yu.A Polkanov, The Shock-Metamorphic Minerals of Carbon, Naukova Dumka Press, Kiev, 1992. 172, (in Russian).

[6] D.J. Kennetta, J.P. Kennett, A. West, G.J. West, T.E. Bunch, B.J. Culleton, J.M. Erlandsona, S.S. Que Hee, J.R. Johnson, C. Merceri, F. Shen, M. Sellers, T.W. Stafford Jr., A. Stich, J.C. Weaver, J.H. Wittke, W.S. Wolbach, PNAS (2009) 12623-12628.
[7] A.W. Phelps, Interstellar diamonds: 1. Condensation and nucleation, Lunar and Planetary science XXX (1999), Abstract 11749 .

[8] G.B. Bokii, G.N. Bezrukov, Ju.A. Kluev, A.M. Naletov, V.I. Nepsha, Natural and Synthetic Diamonds, Nauka, Moscow, 1986. 221, (in Russian).

[9] S.A. Vishnevsky, V.P. Afanasiev, R.P. Argunov, N.A. Palchik, Impact Diamonds: Their Features, Origin and Meaning, SORAN Edition House, United Institute of Geology, Geophysics and Mineralogy, Novosibirsk, 1997. 50, (in Russian).

[10] Z. Pan, H. Sun, Y. Zhang, Ch. Chen, Phys. Rev. Lett. 102 (2009) 055503.

[11] K. Harano, T. Satoh, H. Sumiya, Diamond Relat. Mater. 24 (2012) 78-82.

[12] G. Pratesi, A. Lo Giudice, S. Vishnevsky, C. Manfredotti, C. Cipriani, Am. Mineral. 88 (2003) 1778.

[13] A.M. Zaitsev, Optical Properties of Diamonds, A Data Handbook, Springer-Verlag, Berlin, 2001. 502.

[14] R. Rost, Yu. Dolgov, S.A. Vishnevsky, Dokl. Akad. Nauk SSSR 241 (1978) 695-698, (in Russian).

[15] G. Zimmerer, Nucl. Inst. Methods Phys. Res. A 308 (1991) 178.

[16] M.C. Payne, M.P. Teter, D.C. Allan, T.A. Arias, J.D. Joannopoulos, Rev. Mod. Phys. 64 (1992) 1045.

[17] S.J. Clark, M.D. Segall, C.J. Pickard, P.J. Hasnip, M.J. Probert, K. Refson, M.C. Payne, Z Krystallogr. 220 (2005) 567.

[18] J.S. Lin, A. Qtseish, M.C. Payne, V. Heine, Phys. Rev. B 47 (1993) 4174.

[19] L. Kleinman, D.M. Bylander, Phys. Rev. Lett. 48 (1982) 1425.

[20] H.J. Monkhorst, J.D. Pack, Phys. Rev. B 13 (1976) 5188-5192.

[21] A. Seidl, A. Gorling, P. Vogl, J.A. Majewski, M. Levy, Phys. Rev. B 53 (1996) 3764.

[22] E.D. Palik, Handbook of Optical Constants of Solids; Orlando, Academic Press, New York, 1985.

[23] R.W. Godby, M. Schluter, L.J. Sham, Phys. Rev. B 37 (1988) 10159

C. S. Wang, B. W. Klein, First-principles electronic structure of Si, Ge, GaP, GaAs, $\mathrm{ZnS}$ and ZnSe. 1. Self-consistent energy bands, charge densities and effective masses, Phys. Rev. B 24 (1981) 3417.

[24] S. Baroni, S. de Gironcoli, A. dal Corso, P. Giannozzi, Rev. Mod. Phys. 73 (2001) $515-562$.

[25] D. Porezag, M.R. Pederson, Phys. Rev. B 54 (1996) 7830.

[26] C.D. Clark, in: R. Berman (Ed.), Physical Properties of Diamond, Clarendon Press, Oxford, 1965, p. 443.

[27] F.P. Bundy, J.S. Kasper, J. Chem. Phys. 46 (1967) 3437-3446.

[28] M.E. Straumanis, E.Z. Aka, J. Am. Chem. Soc. 73 (1951) 5643-5646.

[29] M.R. Salehpour, S. Satpathy, Phys. Rev. 41 (N5) (1990) 3048-3052.

[30] B. Wen, J. Zhao, M. Bucknum, P. Yao, T. Li, Diamond Relat. Mater. 17 (2008) 356-364.

[31] N.A. Bakr, A.M. Fundel, V.S. Waman, M.M. Kamble, R.R. Hawaldar, D.P. Amalnerkar, S.W. Gosavi, S.R. Jadkar, Pramana J. Phys. 76 (N3) (2011) 519-531.

[32] Properties of Silicon Carbide (SiC). Ioffe Institute. http://www.ioffe.ru/SVA/NSM/ Semicond/SiC/. Retrieved 2009-06-06.

[33] A. Bakin, in: M. Shur, S. Rumyantsev, M. Levinshtein (Eds.), Chap.:"SiC Homoepitaxy and Heteroepitaxy", Vol. 1, World Scientific, ISBN: 981-256-835-2, 2006, pp. 43-76, (http://www.worldscibooks.com/engineering/6134.html).

[34] H. Sumiya, K. Harano, K. Arimoto, H. Kagi, S. Odake, T. Irifune, Jpn. J. Appl. Phys. 48 (2009) 120206.

[35] A. Ferrari, J. Robetrson, Phil. Trans. Roy. Soc. Lond. A362 (2004) 2477-2512.

[36] S. Reich, C. Thomsen, Phil. Trans. Roy. Soc. Lond. A362 (2004) 2271-2288.

[37] W.A. Yarbrough, R. Messier, Science 247 (1990) 688.

[38] D.S. Knight, W.B. White, J. Mater. Res. 4 (1989) 385-393.

[39] N.B. Reshetnyak, V.A. Ezersky, Mineral. J. 12 (5) (1990) 3-9, (in Russian).

[40] T. Moroz, E. Fedorova, S. Zhmodik, A. Mironov, G. Rylov, A. Ragozin, A. Afanasyev, V. Zaikovsky, Chem. Sustain. Dev. 8 (2000) 43-47.

[41] V.N. Denisov, B.N. Mavrin, N.R. Serebryanaya, G.A. Dubitsky, V.V. Aksenenkov, A.N. Kirichenko, N.V. Kuzmin, B.A. Kulnitskiy, I.A. Perezhogin, V.D. Blank, Diamond Relat. Mater. 20 (2011) 951-953.

[42] T.G. Shumilova, E. Mayer, S.I. Isaenko, Dokl. Earth Sci. 441 (2011) 1552-1554.

[43] C.V. Chiem, H.-K. Seo, S.G. Ansari, G.-S. Kim, J. Seo, H.-S. Shim, Korean J. Chem. Eng. 20 (6) (2003) 1154-1157.

[44] M.H. Grimsditch, E. Anastassakis, M. Cordona, Phys. Rev. B 18 (1997) 901.

[45] http://www.dst.unisi.it/geofluids/raman/spectrum_frame.htm.

[46] J.F. Asell, M. Nicol, J. Chem. Phys. 49 (1968) 5395.

[47] S.-H. Shim, T.S. Duffy, Am. Mineral. 87 (2001) 318-326.

[48] O. Shebanova, The High-Pressure Study on the Fe-O System: Thermodynamics and Phase Transitions of Iron Ferrite $\left(\mathrm{FeFe}_{2} \mathrm{O}_{4}\right)$. , PhD Thesis Uppsala, 2003.

[49] S. Bhargava, H.D. Bist, S. Sahli, M. Aslam, H.B. Tripathi, Appl. Phys. Lett. 67 (1995) 1706.

[50] F. Tuinstra, J.L. Koening, J. Chem. Phys. 53 (1970) 126.

[51] S. Lin, B.J. Feldman, Phys. Rev. Lett. 48 (1982) 829

[52] J. Wagner, P. Lautenschlager, J. Appl. Phys. 59 (1986) 2044.

[53] L. Bergman, B.R. Stoner, K.F. Turner, J.T. Glass, R.J. Nemanich, J. Appl. Phys. 73 (8) (1993) 3951-3957.

[54] S. Sugano, Y. Tanabe, H. Kamimura, Multiplets of Transition Metal Ions in Crystals, Acad. Press, N.Y., 1970 